\title{
BEC-BCS crossover of a trapped Fermi gas without using the local density approximation
}

\author{
R. Jáuregui, R. Paredes, and G. Toledo Sánchez \\ Instituto de Física, Universidad Nacional Autónoma de México, \\ Apdo. Postal 20-364, México D. F. 01000, México.
}

(Dated: April 11, 2018)

\begin{abstract}
We perform a variational quantum Monte Carlo simulation of an interacting Fermi gas confined in a three dimensional harmonic potential. This gas is considered as the precursor system from which a molecular bosonic gas is formed. Based on the results of two-body calculations for trapped atoms, we propose a family of variational many-body wave functions that takes into account the qualitative different nature of the BCS and BEC regimes as a function of the scattering length. Energies, densities and correlation functions are calculated and compared with previous results for homogeneous gases. Universality tests at the unitarity limit are performed including the verification of the virial relation and the evaluation of the $\beta$ parameter.

PACS numbers: 03.75.Ss, 03.75.Hh, 05.30.Fk
\end{abstract}

The crossover from a low interacting attractive Bardeen-Cooper-Schieffer (BCS) gas to a molecular BoseEinstein condensate (BEC) has been realized experimentally using a mixture of ultracold Fermi atoms in two hyperfine states 1, 2, 3, 4, 5]. In dilute Fermi gases, the atomic interactions have a range much smaller than the interparticle separation. Nevertheless, under proper conditions, a magnetic field can be used to tune the attractive potential so that the energy of a pair of scattering atoms is close to that of a molecular bound state (Feshbach resonance). In experiments where the resonance is broad, it can be represented by a single-channel model in which the $s$-wave scattering length $a$ determines the general features of the ultracold atomic gas. At low enough temperatures, atoms in different hyperfine states pair into bound molecules for positive values of $a$, forming a molecular BEC that can be adiabatically converted into a degenerate Fermi gas by shifting $a$ to negative values. At resonance $(|a| \rightarrow \infty)$, the gas acquires universal properties, i. e., they are independent of any feature of the atomic potentials [2, 6, 7]. This is the so called unitarity limit.

The theoretical description of the BEC-BCS crossover is complicated because there is no ad hoc single parameter to control the relevant features in both regimes. A reasonable alternative to infer properties of the system at unitarity has been to consider an homogeneous Fermi gas, and to assume the universality hypothesis according to which the only dominant length is the interparticle separation. Then, the thermodynamic potentials have a universal form specified by only few universal numbers [7]. For instance, the interaction energy is proportional to the Fermi energy via a universal constant $\beta$.

Quantum Monte Carlo (QMC) techniques support $a b$ initio methods to theoretically test the universality hypothesis. They can be used to approximately solve the many-body Schrödinger equation for a given model of the interaction potential. In previous studies the BEC and BCS regimes have been explored using a fixed-node QMC technique, which is rigorous but also computationally de- manding. In particular, the value of $\beta$ has been predicted considering up to 66 particles [8, 9]. Nevertheless, these QMC calculations have been performed for homogeneous gases despite the fact that experimentally the atoms are confined by an external field and therefore the inhomogeneity is intrinsic to the problem. Comparison of QMC results with experiments are then based on a mapping of the trapped system onto a corresponding homogeneous problem with a local value of the Fermi energy $\epsilon_{F}(x)$ (local density approximation). This reasoning also allows to study the universality hypothesis consequences for a trapped dilute Fermi gas in the crossover [2, 10, 11].

Here we study the behavior of a balanced mixture of $2 N$ interacting trapped Fermi atoms, using a variational quantum Monte Carlo (VQMC) technique, which allows to deal with large number of particles but strongly depends on the choice of the variational wave function. We compute the many-body ground state of the inhomogeneous gas from the BCS to the BEC regime for a given short range interaction potential. For simplicity we shall consider an isotropic harmonic potential. The values of $N$ are the highest reported in QMC calculations for this kind of atomic systems. Emphasis will be made on the energies, densities and two point correlation functions. At the unitarity limit, $\beta$ will be directly evaluated and the validity of the virial theorem verified [11].

To describe the many-body system, let us first consider the attractive two-body interaction potential $V\left(r_{i j}\right)=$ $-V_{0} e^{-2\left|\vec{r}_{i}^{\uparrow}-\vec{r}_{j}^{\downarrow}\right| / b}$ of range $b / 2$, to model the effective interaction between atoms in different hyperfine states. The corresponding two-body problem for a particle of reduced mass $m / 2,\left[\hat{p}^{2} / m+V\right] \varphi=E \varphi$, has analytical $s$-wave solutions [12] $\varphi\left(r_{i j}\right)=u\left(r_{i j}\right) / r_{i j}$ both in the continuum $\left(u(y)=c_{1} J_{i b \sqrt{E m} / \hbar}(y)+c_{2} J_{-i b \sqrt{E m} / \hbar}(y)\right)$ and in the bound region $\left(u(y)=c_{+} J_{b \sqrt{|E| m} / \hbar}(y)\right)$. Here $y=\left(b \sqrt{V_{0} m} / \hbar\right) e^{-r / b}$ and $J_{\nu}$ represents the first kind 
Bessel function of order $\nu$. The scattering length is

$$
a=-b\left[\frac{\pi}{2} \frac{N_{0}\left(b \sqrt{V_{0} m} / \hbar\right)}{J_{0}\left(b \sqrt{V_{0} m} / \hbar\right)}-\log \left(b \sqrt{V_{0} m} / 2 \hbar\right)-C\right]
$$

with $N_{0}$ the second kind Bessel function of zero order, and $C$ the Euler constant. This scattering length diverges whenever $J_{0}\left(b \sqrt{V_{0} m} / \hbar\right)=0$. If $z_{n}, n=0,1,2, \ldots$ are the zeros of this Bessel function in increasing order, the potential $V\left(r_{i j}\right)$ admits just $n$-bound states for $z_{n}<b \sqrt{V_{0} m} / \hbar<z_{n+1}$. The discrete eigenvalues are determined by the boundary condition at $r_{i j}=0$, $J_{b \sqrt{|E| m} / \hbar}\left(b \sqrt{V_{0} m} / \hbar\right)=0$. Taking into account the presence of a spherical harmonic trap the Schrödinger equation, $\left[\hat{p}^{2} / m+m \omega^{2} r^{2} / 4+V(r)\right] \varphi(r)=E \varphi(r)$, can be numerically solved and some interesting results are obtained when the range of the potential $b / 2<<\sqrt{\hbar / m \omega}$ [13]. For instance: (i) The ground state energy $E_{0}$ for $b \sqrt{V_{0} m} / \hbar=z_{0}$ (which implies $|a| \rightarrow \infty$ and a zeroenergy resonance in the homogeneous problem) becomes $E_{0} \sim 0.5 \hbar \omega$ as $b \sim 0$ (e.g. for $b=0.03 \sqrt{\hbar / m \omega}$, $\left.E_{0}=0.510655588 \hbar \omega\right)$ while the $s$-wave excited states have energies $\sim\left(2 n_{e}+0.5\right) \hbar \omega ;$;ii) the ground state for $z_{0} \leq b \sqrt{V_{0} m} / \hbar<z_{1}$ can be well approximated by $u(y(r)) \exp \left(-m r^{2} / 2 \hbar \omega\right) / r$. Result (i) is consistent with the analytical solution of the two-body problem in the presence of a contact interaction with a coupling constant determined by the scattering length [14].

To address the many-body system we rely on the VQMC method. A trial wave function $\phi_{T}$ is assumed and, from initial stochastically generated events, a Metropolis Monte Carlo algorithm samples the distribution $\left|\phi_{T}\right|^{2} / \int\left|\phi_{T}\right|^{2}$ for variations of the atoms positions. After a thermalization process, the energy is evaluated at each step. The average energy converges to its expectation value, provided enough points are taken to sample. The parameters used to characterize $\phi_{T}$ are then varied to search a minimum of the energy. The strength of this method relies on the proper choice of the trial wave function. Here, the trial wave function in the region of negative scattering length, $0<b \sqrt{V_{0} m} / \hbar<z_{0}$, has the Jastrow-Slater form

$$
\Psi_{\lambda}(x)=e^{-\lambda \sum_{i, j=1}^{N} V\left(r_{i j}\right)} \Phi_{F G}\left(\sqrt{\omega^{\prime} / \omega} x\right)
$$

where $\lambda$ and $\omega^{\prime}$ are variational parameters, and $\Phi_{F G}(x)$ is the Fermi gas wave function given by a product of Slater determinants (one for each hyperfine state) describing a noninteracting system of harmonically trapped atoms. This variational wave function has the advantage of being exact when no interactions between hyperfine states are allowed $\left(\lambda=0, \omega^{\prime}=\omega\right)$ and does not require an explicit introduction of a healing distance [8, 15]. It is inspired on previous calculations for the nuclear matter [16], where an appropriate choice of the potential allows to explore dynamically the interplay of the nuclear-to-quark matter regime while being exact in both limits. Other forms of the Jastrow wave function can be found in Refs. [8, 9] .
In the region of non-negative scattering length and $z_{0} \leq b \sqrt{V_{0} m} / \hbar<z_{1}$, the Jastrow-Slater wave function over estimates the energy. Therefore, we propose the following trial wave function

$$
\Psi_{\lambda}(x)=\mathcal{A} \phi_{\lambda}\left(1,1^{\prime}\right) \ldots \phi_{\lambda}\left(N, N^{\prime}\right)
$$

where $\mathcal{A}$ is the antisymmetrizer operator and

$$
\phi_{\lambda}(i, j)=u\left(y\left(r_{i j}\right)\right) e^{\left(-m\left|\vec{r}_{i j}\right|^{2} / 4 \hbar \omega\right)} e^{\left(-\lambda m\left|\vec{R}_{i j}\right|^{2} \hbar \omega\right)} / r_{i j}
$$

with $\vec{r}_{i j}$ and $\vec{R}_{i j}$ the relative and center of mass coordinates associated to $\vec{r}_{i}^{\uparrow}$ and $\vec{r}_{j}^{\downarrow} ; \lambda$ is the variational parameter. The structure of this trial wave function is a variational extension for the inhomogeneous gas from that proposed in Ref. [9].

Given $2 N$ particles, a fixed value of $b$ and a scattering length $a$, we determine the energy for a set of values of the variational parameters to pick up the optimal. Each run used about $10^{3}$ steps for thermalization and about $10^{4}$ more to take data. In addition, we estimate the dependence on the initial conditions that might be not erased in the thermalization process. The quoted errors take into account all the above factors.

The results obtained for the optimal energy per particle as a function of the scattering length $a$ are shown in Fig. 1. The values are normalized as described in its caption. By construction these normalized energies take the asymptotic value of zero (one) for small positive (negative) values of $a$. The fact that these energies fall in a curve almost independent of the value of $N$ reflects that the Fermi wave number $k_{F}$, associated to the ideal Fermi gas energy $E_{I F G}=\hbar^{2} k_{F}^{2} / 2 m \approx(6 N)^{1 / 3} \hbar \omega$, defines the proper distance scale to measure $a$. It also means that the same curve can be expected for larger values of $N$ and supports a universal behavior in the crossover.

Notice that in the BCS side of the crossover the interaction energy is very small compared to $E_{I F G}$, in accordance with previous QMC calculations in homogeneous gases [8, 9]. In fact, we have checked that for $-k_{F} a<1$ this variational energy coincides with the result obtained using an effective contact interaction [17]. At unitarity and for $a>0$, the role of the interaction energy becomes more relevant. It is found that in the extreme BEC limit $k_{F} a \rightarrow 0^{+}$there is a strong competition between Pauli blocking effects and the attractive interaction between the atoms in different hyperfine states. This leads to numerical precision problems that could indicate that this highly correlated system should be described beyond the simple scheme of almost non-interacting molecules.

The $\beta$ parameter for a trapped gas is usually evaluated according to the following reasoning [2, 10]. Within the universality hypothesis, for a trapped gas at unitarity, the equation of state would be $(1+\beta) \epsilon_{F}+U_{H O}=\mu_{0}$, with $\epsilon_{F}(x)$ the local Fermi energy, $U_{H O}$ the trapping potential, and $\mu_{0}$ the global chemical potential. This equation is equivalent to that of a trapped noninteracting gas of particles with an effective mass $m /(1+\beta)$ [18] so that the effective chemical potential is also simply scaled 
to $\mu_{0}=E_{I F G} \sqrt{1+\beta}$. The total energy at $T=0$, which determines the energy scale is then:

$$
E_{U}=E_{I F G} \sqrt{1+\beta} .
$$

Since we are working with few atoms it is necessary to take into account the exact expression for $E_{I F G}$. For a closed shell configuration (all single particle states with energies below $E_{F}=\left(\mathcal{M}_{F}+3 / 2\right) \hbar \omega$ are occupied $)$ this energy is given by [17]

$$
E_{I F G} / 2 N \hbar \omega=(3 / 4)\left(\mathcal{M}_{F}+2\right),
$$

instead of the large $N$ limit, $E_{I F G} / 2 N=3 E_{F} / 4$, which produces a slightly underestimated value.

The universality hypothesis can be tested independently through a virial relation [11] resulting from mechanical equilibrium conditions on the trapped unitary gas. According to it, for our system

$$
E_{U} / 2=2 N\left\langle m \omega^{2} R^{2} / 2\right\rangle .
$$

In Table 1 we show the energy per particle at unitarity $E_{U} / 2 N$ obtained from the VQMC calculation and the trap energy $m \omega^{2}\left\langle R^{2}\right\rangle$ for closed shell configurations with $\mathcal{M}_{F} \leq 9$. It can be noticed that, within error bars, the virial relation Eq.(7) is satisfied. The approximate linear behavior of $E_{U} / 2 N$ as a function of $\mathcal{M}_{F}$ :

$$
E_{U} / 2 N \hbar \omega \sim 0.53 \pm 0.01\left(\mathcal{M}_{F}+1.95 \pm 0.06\right)
$$

supports the universality relationship Eq. (5) with the universal parameter $\beta=-0.50_{-0.04}^{+0.02}$.

Previous theoretical calculations predict $\beta \sim-0.326$ [6], $\beta \sim-0.4$ [18, 19], $\beta \sim-0.56$ [8, 9], $\beta=-0.75$ [20], $\beta=-0.492$ [21] and $\beta=-0.545$ [22]. The first experimental measurements yielded $\beta \sim-0.3$ [4], $\beta=-0.49 \pm$ 0.04 [10], $\beta=-0.64 \pm 0.15$ [5] and $\beta=-0.68_{-0.1}^{+0.13}[3]$. More recently the value $\beta=-0.54 \pm 0.05[23$ ] and $\beta=$ $-0.54_{-0.12}^{+0.05}$ 24] was found for ${ }^{6} \mathrm{Li}$ and ${ }^{40} \mathrm{~K}$ respectively.

The single-particle and the two-particle correlation functions were calculated as a function of the scattering length. First, we analyze the density profile as a function of the distance to the center of the harmonic trap (Fig. 2). The numerical profile of the ideal gas reproduces satisfactorily the Thomas-Fermi (TF) prediction. At unitarity, the density resembles more a TF than a Gaussian profile, as predicted in Ref. [25]. For the BEC regime a clear molecular bunching is observed around the origin, however, due to molecular repulsion, these bosonic molecules are prevented from collapsing to the center of the trap.

By analyzing the two point correlation functions, Pauli blocking can be observed. As expected, the radii at which no atoms of the same species can be found diminishes as the intensity of the interaction increases for a given range of the potential [13]. In Fig. 3 we compare the two point correlation functions $K\left(\vec{r}_{i}^{\uparrow}, \vec{r}_{j}^{\downarrow}\right)$ of atoms in different hyperfine states in the ideal and BEC regimes. The enhancement of correlation for short $r_{i j}$ near the center of the trap $\left(R_{c m}<0.65 \sqrt{\hbar / m \omega}\right)$ indicates molecule formation, while the increase of probability of finding pairs of particles separated at relative distances of the order of 1.3 and $2.3 \sqrt{\hbar / m \omega}$ with center of mass radii 0.65 and $1.09 \sqrt{\hbar / m \omega}$ is a manifestation of molecular condensation. A similar analysis performed for the BCS regime shows a slight increase of long distance correlations of atoms in different states.

Summarizing, we have performed for the first time direct tests of the universality hypothesis in the unitarity limit for an inhomogeneous interacting Fermi gas using VQMC techniques. These tests include: the $N$-independent energy curve features as described in Fig. 1, the verification of virial relations for each $N$ and the variational evaluation of $\beta$ using a linear fit of the energy per particle $E_{U} / 2 N$ as a function of the Fermi number $\mathcal{M}_{F}$. We have also shown that the optimized wave functions yield reasonable values compared with experimental observations not just for the energy per particle, but also for the mean radii at the different regimes, the densities and the two point correlation functions.

Acknowledgments. This work was partially supported by Conacyt 41048-A1 and DGAPA-UNAM contract PAPIIT IN117406-2.
[1] S. Jochim et al., Science 302, 2101 (2003); M. Greiner, C. A. Regal and D. S. Jin, Nature 426, 537 (2003); M. W. Zwierlein et al., Phys. Rev. Lett. 91, 250401 (2003); K. E. Strecker, G. B. Partridge and R. G. Hulet, Phys. Rev. Lett. 91, 080406 (2003).

[2] K. M. O'Hara et al., Science 298, 2179(2002); M. E. Gehm et al., Phys. Rev. A 68, 011401(R) (2003).

[3] M. Bartenstein et al., Phys. Rev. Lett. 92, 120401 (2004).

[4] T. Bourdel et al., Phys. Rev. Lett. 91, 020402 (2003).

[5] T. Bourdel et al., Phys. Rev. Lett. 93, 050401 (2004).

[6] H. Heiselberg, Phys. Rev. A 63, 043606 (2001).

[7] T.-L. Ho, Phys. Rev. Lett. 92, 090402 (2004).

[8] J. Carlson et al., Phys. Rev. Lett. 91, 050401 (2003); S.
Y. Chang et al., Phys. Rev. A 70, 043602 (2004).

[9] G. E. Astrakharchik et al., Phys. Rev. Lett. 93, 200404 (2004).

[10] J. Kinast et al., Science 307, 1296 (2005).

[11] J. E. Thomas, J. Kinast, and A. Turlapov, Phys. Rev. Lett. 95, 120402 (2005); J. Joseph et al., Phys. Rev. Lett. 98, 170401 (2007).

[12] W. Rarita and R. D. Present, Phys. Rev. 51, 788 (1937).

[13] R. Jáuregui, L.E. Rosales-Zárate, R. Paredes, and G. Toledo Sánchez, in preparation.

[14] T. Busch et al., Foundations of Phys. 28, 549 (1998).

[15] V. R. Pandharipande and H. A. Bethe, Phys. Rev. C 7, 1312 (1973). 
[16] C. J. Horowitz, E. J. Moniz and J. W. Negele, Phys. Rev. D 31, 1689 (1985); C. J. Horowitz and J. Piekarewicz, Nucl. Phys. A 536, 669 (1992); G. Toledo Sánchez, and J. Piekarewicz, Phys. Rev. C 65, 045208 (2002).

[17] R. Jáuregui, R. Paredes, and G. Toledo Sánchez, Phys. Rev. A 69, 013606 (2004).

[18] G. A. Baker, Phys. Rev. C 60, 054311 (1999).

[19] J. R. Engelbrecht, M. Randeria and C. A. R. Sá de Melo, Phys. Rev. B, 55, 15153 (1997).

[20] D. Lee, Phys. Rev. B 73, 115112 (2006).

[21] J. von Stecher and C. H. Greene, Phys. Rev. A 75, 022716 (2007).

[22] A. Perali, P. Pieri, and G. C. Strinati, Phys. Rev. Lett. 93, 100404 (2004).

[23] G. B. Patridge et al., Science 311, 503 (2005).

[24] J. T. Stewart et al., Phys. Rev. Lett. 97, 220406 (2006).

[25] J. Stajic, Q. Chen, and K. Levin, Phys. Rev. Lett. 94, 060401 (2005).

\begin{tabular}{|c|c|c|c|c|c|c|}
\hline $\mathcal{M}_{F}$ & $N$ & $\begin{array}{c}\lambda_{\text {opt }} \\
( \pm 0.005)\end{array}$ & $\begin{array}{c}E_{I F G} / 2 N \\
{[\hbar \omega]}\end{array}$ & $\begin{array}{c}E_{U} / 2 N \\
{[\hbar \omega]}\end{array}$ & $\begin{array}{c}\beta \\
( \pm 0.02)\end{array}$ & $\begin{array}{c}m \omega^{2}\left\langle R^{2}\right\rangle \\
{[\hbar \omega]}\end{array}$ \\
\hline 0 & 1 & 1 & 1.5 & 1 & -0.5556 & $0.99 \pm 0.07$ \\
1 & 4 & 0.666 & 2.25 & $1.32 \pm 0.05$ & -0.66 & $1.44 \pm 0.11$ \\
2 & 10 & 0.472 & 3 & $2.00 \pm 0.05$ & -0.56 & $1.93 \pm 0.16$ \\
3 & 20 & 0.382 & 3.75 & $2.48 \pm 0.05$ & -0.56 & $2.55 \pm 0.10$ \\
4 & 35 & 0.333 & 4.5 & $3.13 \pm 0.05$ & -0.52 & $3.01 \pm 0.12$ \\
5 & 56 & 0.261 & 5.25 & $3.62 \pm 0.05$ & -0.52 & $3.57 \pm 0.14$ \\
6 & 84 & 0.242 & 6 & $4.25 \pm 0.1$ & -0.50 & $4.23 \pm 0.16$ \\
7 & 120 & 0.187 & 6.75 & $4.85 \pm 0.1$ & -0.48 & $4.77 \pm 0.18$ \\
8 & 165 & 0.186 & 7.5 & $5.25 \pm 0.1$ & -0.51 & $5.32 \pm 0.20$ \\
9 & 220 & 0.168 & 8.25 & $5.82 \pm 0.1$ & -0.50 & $5.88 \pm 0.22$ \\
\hline
\end{tabular}

TABLE I: Optimized variational parameter $\lambda$, variational values of the energy per particle $E_{U} / 2 N$ and the trap energy $m \omega\left\langle R^{2}\right\rangle$ for closed shells as a function of $\mathcal{M}_{F}$ at unitarity. The error bars in the last case take into account statistical sources and uncertainties in the value of $\lambda$. The interaction potential range $b / 2$ is varied so that $k_{F} b / 2=0.01$.

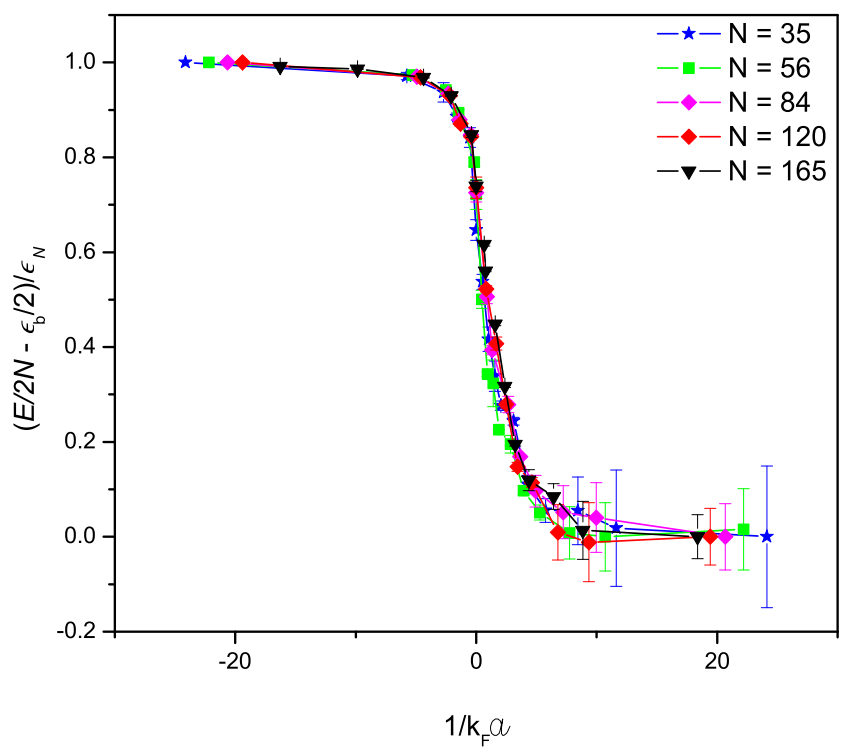

FIG. 1: Variational energy per particle $E / 2 N$ as a function of $1 / k_{F} a$. The normalization factor $\epsilon_{N}$ depends on the number of particles and is defined as follows. The ideal gas energy per particle, $E_{I F G} / 2 N$, is the asymptotic value of the energy per particle for small negative scattering lengths $E\left(a_{-}\right) / 2 N$; the asymptotic value $E\left(a_{+}\right) / 2 N$ corresponds to the energy per particle for small positive scattering lengths (here $a_{+}=0.012 \sqrt{\hbar / m \omega}$ ) minus half the bound molecular energy $\tilde{\epsilon}_{b}\left(a_{+}\right) / 2$ for this value of $a$ (here $\left.\tilde{\epsilon}_{b}\left(a_{+}\right)=2540.22 \hbar \omega\right)$. The $N$ dependent normalization factor $\epsilon_{N}$ is the difference between $E\left(a_{-}\right)$and $E\left(a_{+}\right)$. Finally $\epsilon_{b}(a) / 2=\tilde{\epsilon}_{b}(a) / 2+E\left(a_{+}\right) / 2 N$. In these calculations the range of the interactions is $b / 2=0.015 \sqrt{\hbar / m \omega}$. 




FIG. 2: Density profiles for $2 N=330$ atoms. The range of the potential is $b / 2=0.015 \sqrt{\hbar / m \omega}$ and the scattering length for the BEC density corresponds to $1 / k_{F} a=3.25$.

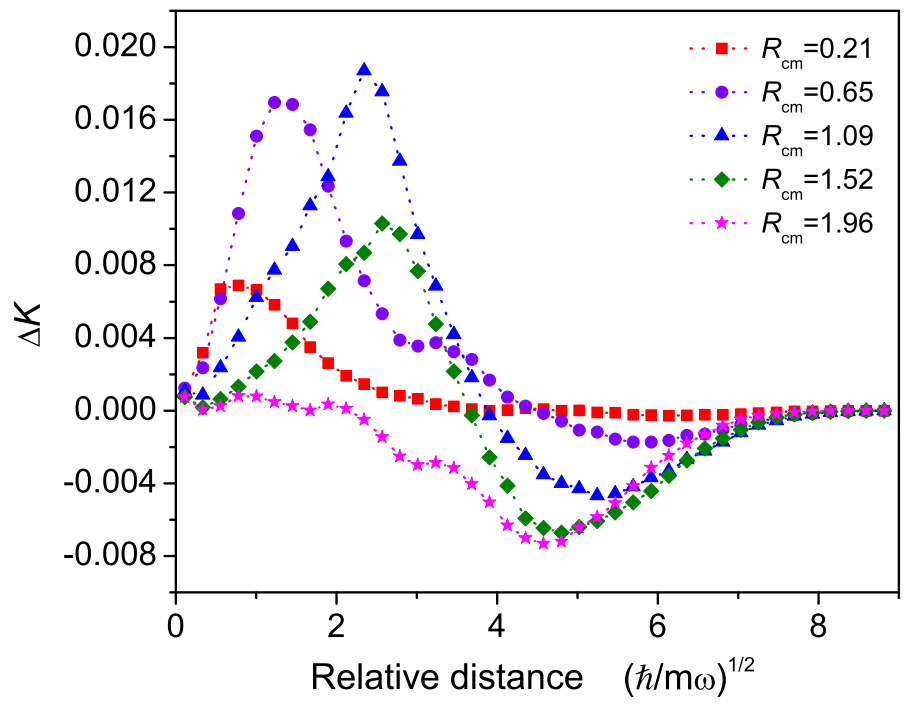

FIG. 3: Probability difference $\Delta K$ that two particles with antiparallel spin are found separated a distance $r_{i j}$ in the BEC and ideal regimes. Each curve in this figure correspond to different spherical radii $R_{c m}$ measured from the center of the trap. Calculations are performed at $b / 2=0.015 \sqrt{\hbar / m \omega}$ and $1 / k_{F} a=3.25$. 